\title{
A Cost-Effectiveness Analysis Of Pregabalin For The Treatment Of Patients With Chronic Cervical Pain With A Neuropathic Component In Japan
}

This article was published in the following Dove Press journal: Journal of Pain Research

\author{
Manabu Akazawa (D) \\ Ataru Igarashi ${ }^{2}$ \\ Nozomi Ebata $\mathbb{1 D}^{3}$ \\ Tatsunori Murata ${ }^{4}$ \\ Shigeki Zeniya (iD) ${ }^{4}$ \\ Yuri Haga ${ }^{5}$ \\ Kazutaka Nozawa ${ }^{3}$ \\ Koichi Fujii ${ }^{3}$ \\ Toshihiko Taguchi ${ }^{6}$
}

'Public Health and Epidemiology, Meiji Pharmaceutical University, Tokyo, Japan; ${ }^{2}$ Health Economics and Outcomes Research, Graduate School of Pharmaceutical Sciences, The University of Tokyo, Tokyo, Japan; ${ }^{3}$ Medical Affairs, Pfizer Japan Inc., Tokyo, Japan; ${ }^{4}$ CRECON Medical Assessment Inc., Tokyo, Japan; ${ }^{5}$ Clinical Research Division, Clinical Study Support, Inc., Nagoya, Japan; ${ }^{6}$ Yamaguchi Rosai Hospital, Yamaguchi, Japan
Correspondence: Nozomi Ebata

Medical Affairs, Pfizer Japan Inc., 3-22-7

Yoyogi, Shibuya-ku, Tokyo I5I-8589 Japan

Tel +8I 38033930745

$\mathrm{Fax}+81353099198$

Email nozomi.ebata@pfizer.com
Purpose: To evaluate the cost-effectiveness of pregabalin versus other analgesics among patients with chronic cervical pain with neuropathic components during routine clinical practice in Japan.

Patients and methods: The analysis considered patients with chronic cervical pain with a neuropathic pain component (radiating pain to the upper limb) and who were treated with pregabalin with or without other analgesics (pregabalin-containing treatments) or other analgesics alone (usual care) for 8 weeks. Other analgesics included non-steroidal antiinflammatory drugs (NSAIDs), weak opioids, antidepressants, and antiepileptic drugs. A Markov cohort simulation model was constructed to estimate costs and effectiveness (in terms of quality-adjusted life-years, QALYs) of each treatment over a 12-month time horizon. In the model, patients transitioned among three states of pain severity (no/mild, moderate, and severe). Data were derived from a previous observational study (pregabalincontaining treatments, $\mathrm{n}=138$; usual care, $\mathrm{n}=211$ ). Cost inputs included medical costs and productivity losses. QALYs were calculated using the EuroQol five-dimensional, five-level questionnaire. The cost-effectiveness was evaluated using incremental cost-effectiveness ratios (ICERs). Sensitivity analyses were conducted to assess the robustness of results.

Results: From the payer's perspective, pregabalin-containing treatments were more costly (JPY 61,779 versus JPY 26,428) but also more effective (0.763 QALYs versus 0.727 QALYs) than the usual care, with an ICER of JPY 970,314 per QALY gained. From the societal perspective, which also included productivity losses, the ICER reduced to JPY 458,307 per QALY gained. One-way sensitivity analyses demonstrated the robustness of the results. Given a hypothetical threshold value of one additional QALY of JPY 5,000,000, the probability of pregabalin-containing treatments being cost-effective was $100 \%$.

Conclusion: Compared with using other analgesics alone, the use of pregabalin, alone or in addition to other analgesics, was cost-effective for the treatment of chronic cervical pain with a neuropathic pain component in Japan.

Keywords: neuropathic pain, quality-adjusted life-year, incremental cost-effectiveness ratio, health economics

\section{Introduction}

Chronic neck pain is a common global health problem, with an estimated prevalence of 358 million people in 2015. ${ }^{1}$ According to the Global Burden of Disease Study 2015, low back and neck pain was the leading global cause of disability. ${ }^{1}$ Neck pain is also common among Japanese people, with the prevalence of neck and shoulder pain being $48.3 \%{ }^{2}$ Among people with chronic musculoskeletal pain in 
Japan, the neck was the second most common site of pain $(55 \%)$ after the lower back (65\%), and about $20 \%$ of people with chronic pain reported the neck as the site of most persistent pain. ${ }^{3}$

Different pathophysiological mechanisms can categorize neck pain as nociceptive, neuropathic, or secondary to another cause. ${ }^{4}$ Among them, neuropathic pain $(\mathrm{NeP})$ causes great burden; for example, patients with chronic NeP have more severe pain than those with other chronic pain, ${ }^{5}$ experience poor quality of life (QOL), and have high healthcare costs. ${ }^{6}$ Common neck pain conditions that involve $\mathrm{NeP}$ include cervical radiculopathy, cervical myelopathy, and cervical disc herniation. Although no data are available on the prevalence of chronic neck pain with $\mathrm{NeP}$ components, one study previously reported that approximately half of neck pain patients unresponsive to previous treatment had at least some NeP components. ${ }^{7}$ To lessen the burden on these patients, appropriate treatment should be provided to patients with neck pain with a NeP component.

Pregabalin, a ligand for the $\alpha_{2}-\delta$ subunit of voltagegated calcium ion channels, is recommended as a first-line medication in the pharmacotherapy for $\mathrm{NeP}^{8-10}$ In the US, pregabalin is indicated only for specific pain conditions (that is, NeP associated with diabetic peripheral neuropathy or spinal cord injury, postherpetic neuralgia, and fibromyalgia). ${ }^{11}$ However, in Japan where the indication of pregabalin was expanded to $\mathrm{NeP}$ in $2013,{ }^{12}$ it can be used for patients with $\mathrm{NeP}$ associated with cervical radiculopathy or myelopathy. Pregabalin is also widely used for $\mathrm{NeP}$ treatment in the $\mathrm{EU}$, where it has broad indications for peripheral and central $\mathrm{NeP}$ in adults. ${ }^{13}$

We previously examined the effectiveness of pregabalin for patients with chronic cervical pain with a NeP component in a Japanese clinical setting. ${ }^{14}$ In that observational study, patients who recently initiated pregabalin treatment, with or without other analgesics, reported a significant improvement in pain and sleep compared with those who continued to be treated only with conventional analgesics (such as non-steroidal anti-inflammatory drugs [NSAIDs] and weak opioids). The results showed the clinical benefits of pregabalin for the management of this $\mathrm{NeP}$ condition. However, the study seemed to reveal that many patients with this condition are treated with only conventional analgesics like NSAIDs in real-world clinical settings in Japan, which is against guideline recommendations. ${ }^{10}$

In Japan, pregabalin treatment was shown to be a costeffective treatment for peripheral $\mathrm{NeP}^{15}$ and chronic low back pain with a NeP component. ${ }^{16}$ However, to our knowledge, the cost-effectiveness of pregabalin for chronic neck pain with a NeP component has not yet been evaluated in Japan or any other nation. As its effectiveness has already been shown, ${ }^{14}$ if evidence reveals that using pregabalin is also a cost-effective treatment for this NeP condition, it may help facilitate the use of pregabalin for the management of these patients. Therefore, in this analysis, we evaluated whether using pregabalin, alone or in addition to other analgesics, is cost-effective for the treatment of patients with chronic cervical pain with a NeP component, using data from the above-mentioned observational study. ${ }^{14}$

\section{Methods}

Our cost-effectiveness analysis used a Markov cohort simulation model to estimate costs and effectiveness (in terms of quality-adjusted life-years, QALYs) of pregabalin with or without other analgesics (pregabalin-containing treatments) versus other analgesics only (usual care) for the treatment of chronic cervical pain with a NeP component, over a 12-month time horizon. Other analgesics used in usual care included NSAIDs, weak opioids, antidepressants, and antiepileptic drugs. The cost-effectiveness of pregabalin was evaluated using the incremental cost-effectiveness ratio (ICER) from the healthcare payer's perspective and also from the societal perspective, with the latter including productivity losses. This analysis was based on data taken from a previously conducted study (details provided below), which received ethical approval from the Byoin-Godo Ethical Review Board. Ethical approval was not obtained for the present cost-effectiveness analysis because it was an analysis that used only existing data without involving any new data collection.

\section{Model Structure}

A Markov model was constructed to simulate the course of a cohort for a 12-month time horizon, with a 1-month cycle. The model had three health states describing the level of pain severity, which was determined based on the numerical rating scale (NRS) scores: no/mild pain (score $0-3$ ), moderate pain (4-6), and severe pain (7-10). At the start of the model, patients began with a state of either moderate or severe pain. Patients then remained in the severity category or transitioned to another category at monthly intervals for the initial two months, according to transition probabilities. From month 3 onwards, patients remained in the same severity category as that at the end of month 2 (Figure 1). In the model, treatment discontinuation was assumed to occur in month 1 . Once treatment 


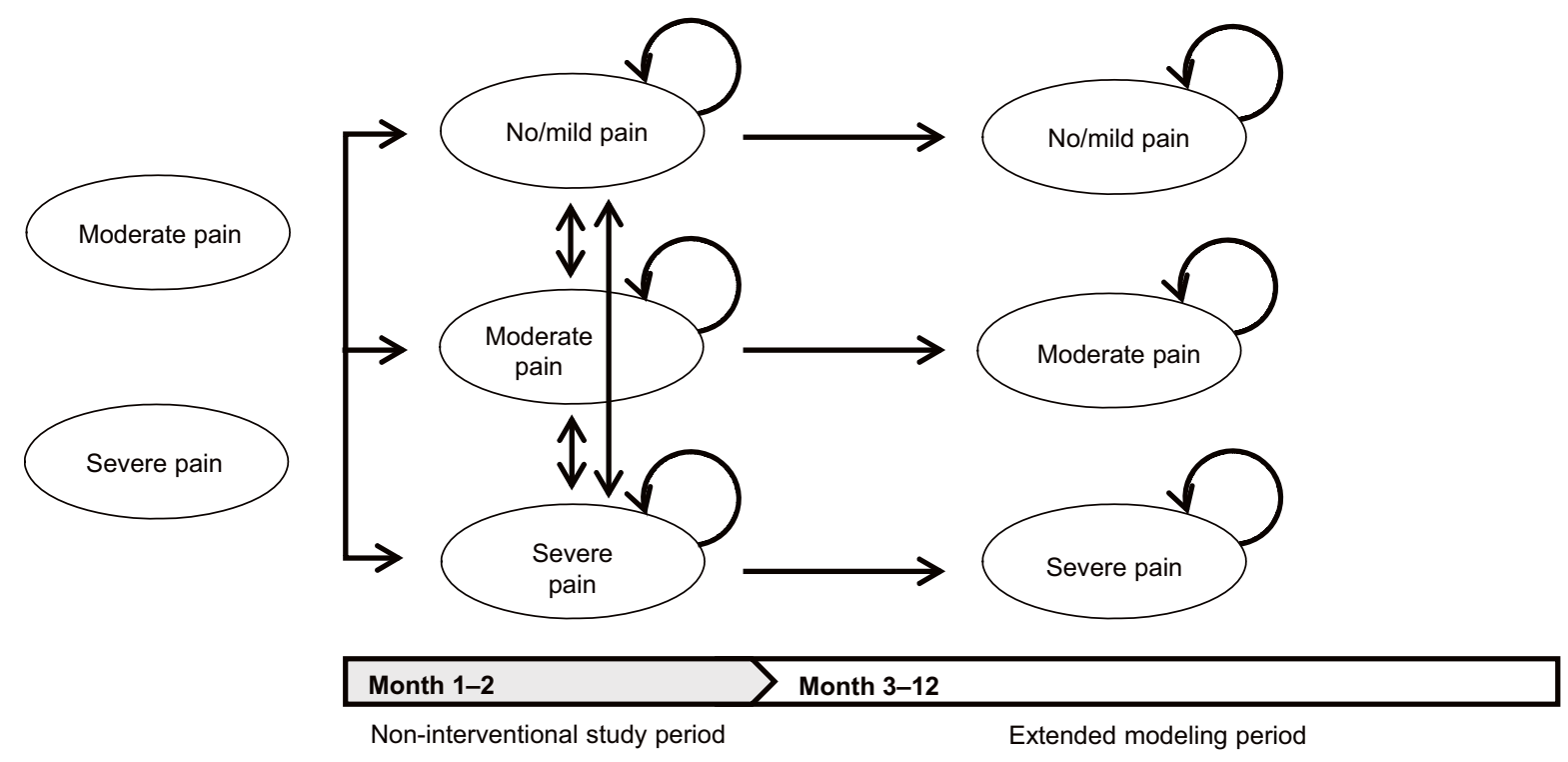

Figure I Framework of the Markov model used for analysis. Pain severity categories were determined based on a numerical rating scale (NRS) score for pain severity (range: 0 to 10). Three severity categories were defined as follows: no/mild pain (NRS score $0-3$ ), moderate pain (4-6), and severe pain (7-10).

was discontinued, the pain NRS score was modeled to revert to the score at baseline. Then, the score was maintained on the conservative assumption that the treatment was no longer effective. Using the constructed model, costs and QALYs were estimated for each treatment group. Due to the short time horizon (12-month), this analysis did not consider a discount for estimation of costs and QALYs.

In the model, the pain severity category at the end of month 2 was maintained for the extended period (months $3-$ 12), based on Japanese long-term studies showing that improved pain scores achieved within 8 weeks of treatment with pregabalin were sustained for the study period over 52 weeks. ${ }^{17,18}$ The 12-month time horizon was chosen as the target disease is a chronic condition and patients can be treated with pregabalin for over a year. This time horizon was also used in previous cost-effectiveness analyses of pregabalin for NeP in Japan. ${ }^{15,16}$ In the present analysis, the model did not assume the potential for patients undergoing surgery because of its low probability. Surgery may be considered when patients have progressive symptoms such as gait disorders or impaired hand dexterity, or when conservative management strategies are not successful. However, there is no established consensus on the indications for surgery in patients with cervical spondylotic myelopathy and cervical radiculopathy, ${ }^{19-21}$ and a conservative therapy is usually chosen, especially for patients with mild symptoms or older patients. Furthermore, this analysis included patients exhibiting stable conditions who sought medical care in a primary care setting. Thus, we expected that the possibility of surgery would be fairly low among patients included in this analysis.

\section{Data}

Data were obtained from our previous non-interventional observational study in a Japanese primary care setting. ${ }^{14}$ Patients were those aged $\geq 20$ years with chronic $(\geq 12$ weeks) cervical pain with pain radiating to the upper limb, and pain refractory to conventional analgesics for $\geq 12$ weeks, as well as with pain severity, self-rated as $\geq 5$ on a NRS. Patients who had been treated with pregabalin within the previous 12 weeks and patients regularly treated with nerve blocks were excluded. Patients were treated in routine clinical practice for 8 weeks, and received either pregabalin-containing treatments (pain treatment with pregabalin with or without other analgesics) or usual care (pain treatment with conventional analgesics such as NSAIDs, weak opioids, antidepressants, and antiepileptic drugs only). As a non-interventional study, all treatment decisions were made at the discretion of physicians, and the choices of pain medications were made independently of their decisions to enroll the patients in the study.

Of the 369 patients enrolled, 145 received pregabalincontaining treatments, and 224 continued the usual care. The pregabalin group contained more male and younger patients than the usual care group (proportion of male, 53.1\% versus $30.4 \%$; mean age, 58.3 years versus 66.4 years). At baseline, patients in the pregabalin group had a shorter pain duration 
(mean \pm standard deviation (SD) duration, $28.8 \pm 38.6$ months versus $37.1 \pm 50.9$ months) and slightly higher pain NRS scores (mean \pm SD scores, $6.1 \pm 1.2$ versus $5.8 \pm 1.1$ ). Pain medications used among the usual care group at baseline were mainly NSAIDs ( $89.3 \%$ of patients used), which were also commonly used among patients in the pregabalin group (93.1\% of patients used at baseline).

Of these 369 patients, 20 patients who dropped out or discontinued treatment for reasons other than adverse events were excluded from data extraction for this costeffectiveness analysis. Patients who discontinued treatment for adverse events were included in data extraction, which was to evaluate the cost-effectiveness considering the unfavorable impacts of treatment. Consequently, this analysis included 138 patients in the pregabalin group and 211 patients in the usual care group (Figure 2). Among these, seven in the pregabalin group and one in the usual care group discontinued treatment because of adverse events.

\section{Model Inputs}

Pain Severity And Transition Probabilities

The initial distribution of severity categories was calculated based on pain NRS scores at baseline among all patients irrespective of treatment group as follows: moderate pain (77.1\%) and severe pain (22.9\%). Transition probability for the initial two months was derived from the actual transition between pain severity categories from week 0 to week 4 (for month 1) and from week 4 to week 8 (for month 2) for each treatment group in the observational study. The discontinuation rates were also derived from the actual rates of discontinuation due to adverse events, and set as $5.1 \%$ for the pregabalin group and $0.5 \%$ for the usual care group. Table 1 shows all the model inputs used in the model, including probability inputs.

\section{Costs (Medical Costs And Productivity Losses)}

In this cost-effectiveness analysis, the evaluation from the healthcare payer's perspective considered only medical costs and the evaluation from the societal perspective considered both medical costs and productivity losses. Costs were expressed in Japanese Yen (JPY) (JPY $100=$ USD 0.89, as of September 20, 2018).

Medical costs considered included costs of pregabalin, other pain medications, and physical therapy (Table 1), and these costs were calculated based on data obtained in the observational study. For pain medications other than

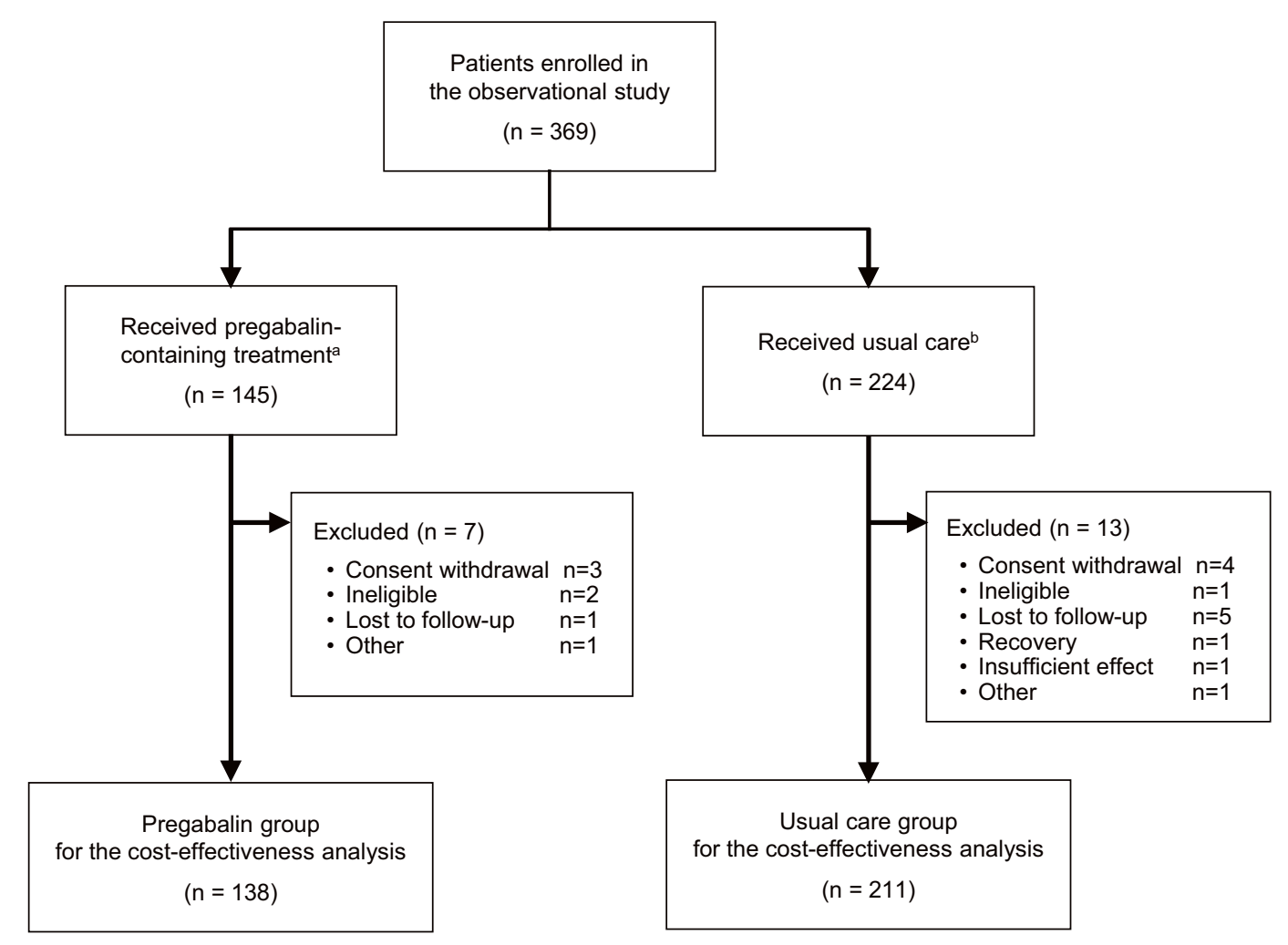

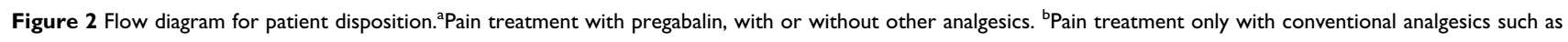
non-steroidal anti-inflammatory drugs (NSAIDs), weak opioids, antidepressants, and antiepileptic drugs. 
Table I Summary Of Model Input Data

\begin{tabular}{|c|c|c|}
\hline Parameter & Value & Range \\
\hline \multicolumn{3}{|l|}{ Cost inputs } \\
\hline \multicolumn{3}{|l|}{ Pregabalin costs (JPY) } \\
\hline Moderate pain, month I & 3,635 & $3,222-4,073$ \\
\hline Severe pain, month I & 4,866 & $3,911-5,924$ \\
\hline No/mild pain, month $2^{\mathrm{a}}$ & 2,924 & $\mathrm{I}, 852-4,237$ \\
\hline Moderate pain, month $2^{\mathrm{a}}$ & 3,538 & $2,476-4,787$ \\
\hline Severe pain, month $2^{\mathrm{a}}$ & 6,587 & $4,20 I-9,502$ \\
\hline \multicolumn{3}{|l|}{ Other pain medication costs (JPY) } \\
\hline Pregabalin group, month I & 923 & $75 I-1,113$ \\
\hline Pregabalin group, month $2^{\mathrm{a}}$ & 1,083 & $88 I-1,306$ \\
\hline Usual care group, month I & 1,109 & $903-1,337$ \\
\hline Usual care group, month $2^{\mathrm{a}}$ & 1,183 & $963-1,426$ \\
\hline \multicolumn{3}{|l|}{ Physical therapy costs (JPY) } \\
\hline Pregabalin group, month I & 780 & $634-940$ \\
\hline Pregabalin group, month $2^{\mathrm{a}}$ & 764 & $622-921$ \\
\hline Usual care group, month I & 1,049 & $854-1,264$ \\
\hline Usual care group, month $2^{\mathrm{a}}$ & 1,045 & $850-1,260$ \\
\hline \multicolumn{3}{|l|}{ Productivity losses (absenteeism + presenteeism) (JPY) } \\
\hline No/mild pain & 52,807 & - \\
\hline Moderate pain & 55,036 & - \\
\hline Severe pain & 68,284 & - \\
\hline \multicolumn{3}{|l|}{ Probability inputs } \\
\hline \multicolumn{3}{|l|}{ Initial distribution probability } \\
\hline Moderate pain & 0.771 & $0.725-0.813$ \\
\hline Severe pain & 0.229 & $0.187-0.275$ \\
\hline \multicolumn{3}{|l|}{ Discontinuation rate } \\
\hline Pregabalin group & 0.051 & $0.021-0.093$ \\
\hline Usual care group & 0.005 & $0.000-0.017$ \\
\hline \multicolumn{3}{|l|}{ Transition probability in pregabalin group } \\
\hline From week 0 to week 4 , moderate pain to no/mild pain & 0.293 & $0.208-0.386$ \\
\hline From week 0 to week 4 , moderate pain to severe pain & 0.061 & $0.023-0.115$ \\
\hline From week 0 to week 4 , severe pain to no/mild pain & 0.139 & $0.048-0.267$ \\
\hline From week 0 to week 4 , severe pain to moderate pain & 0.611 & $0.449-0.761$ \\
\hline From week 4 to week 8 no/mild pain to moderate pain & 0.094 & $0.020-0.214$ \\
\hline From week 4 to week 8 , moderate pain to no/mild pain & 0.267 & $0.180-0.365$ \\
\hline From week 4 to week 8 , moderate pain to severe pain & 0.035 & $0.007-0.082$ \\
\hline From week 4 to week 8 , severe pain to no/mild pain & 0.333 & $0.128-0.581$ \\
\hline From week 4 to week 8 , severe pain to moderate pain & 0.267 & $0.084-0.508$ \\
\hline \multicolumn{3}{|l|}{ Transition probability in usual care group } \\
\hline From week 0 to week 4 , moderate pain to no/mild pain & 0.089 & $0.051-0.137$ \\
\hline From week 0 to week 4 , moderate pain to severe pain & 0.054 & $0.025-0.092$ \\
\hline From week 0 to week 4 , severe pain to no/mild pain & 0.095 & $0.027-0.199$ \\
\hline From week 0 to week 4 , severe pain to moderate pain & 0.238 & $0.124-0.376$ \\
\hline From week 4 to week 8 , no/mild pain to moderate pain & 0.263 & $0.097-0.476$ \\
\hline From week 4 to week 8 , no/mild pain to severe pain & 0.053 & $0.001-0.185$ \\
\hline From week 4 to week 8 , moderate pain to no/mild pain & 0.110 & $0.066-0.164$ \\
\hline From week 4 to week 8 , moderate pain to severe pain & 0.052 & $0.023-0.092$ \\
\hline
\end{tabular}

(Continued) 
Table I (Continued).

\begin{tabular}{|l|l|l|}
\hline Parameter & Value & Range \\
\hline From week 4 to week 8, severe pain to no/mild pain & 0.027 & $0.001-0.097$ \\
From week 4 to week 8, severe pain to moderate pain & 0.378 & $0.231-0.538$ \\
\hline Utility inputs & & \\
Utility of no/mild pain & 0.854 & $0.651-0.975$ \\
Disutility between no/mild pain and moderate pain & 0.116 & $0.092-0.143$ \\
Disutility between moderate pain and severe pain & 0.116 & $0.092-0.143$ \\
\hline Others & & $0.00-0.04$ \\
Discount rate & 0.02 & \\
\hline
\end{tabular}

Notes: JPY $100=$ USD $0.89 .{ }^{a}$ Costs at month 3 to month 12 were assumed to be the same as those at month 2.

pregabalin, the following four classes were considered, and costs were calculated using the prices of drugs shown in parentheses, each of which was chosen as the representative drug of the respective classes in Japan: NSAIDs (loxoprofen sodium hydrate), antidepressants (duloxetine hydrochloride), antiepileptic drugs (gabapentin), and weak opioids (tramadol hydrochloride and tramadol hydrochloride/acetaminophen) (each drug cost per day is provided in Table S1). Costs of physical therapy were calculated according to the medical fee points $(1$ point $=$ JPY 10). A list of physical therapy procedures and respective medical fee points is provided in Table S2.

The health-related work productivity losses were assessed with the Work Productivity and Activity Impairment Questionnaire: General Health V2.2 (WPAI: GH) in the observational study. The WPAI: GH is a 6-item questionnaire that quantitatively assesses both absenteeism (workdays missed) and presenteeism (reduced productivity while at work) due to health problems. ${ }^{22}$ This instrument is considered suitable for direct translation of productivity losses into a monetary figure. ${ }^{23}$ Absenteeism and overall productivity losses (both absenteeism and presenteeism) were estimated using the formula provided by Lofland et al. ${ }^{23}$ The amount of wages used for calculation were based on mean monthly income in Japan. ${ }^{24}$ The absolute levels of absenteeism were low in all severity categories (rate of work time lost due to absenteeism were: $1.0 \%, 0.2 \%$, and $0.03 \%$ in no/mild, moderate, and severe pain, respectively), and patients with severe pain had the lowest absenteeism (mean \pm SD costs, JPY $149 \pm 942$ ). In contrast, the levels of overall productivity losses were higher in patients with more severe pain $(18.9 \%, 20.1 \%$, and $24.5 \%$ in no/mild, moderate, and severe pain, respectively), with patients with severe pain having the highest overall productivity losses (JPY 68,284 $\pm 110,810$ ). As absenteeism was not likely to be a main driver for productivity losses, the present analysis considered the costs of overall productivity losses for the evaluation from the societal perspective. The cost values used in the model are shown in Table 1.

\section{Utility}

The EuroQol five-dimensional, five-level (EQ-5D-5L) index values, ${ }^{25}$ derived by the conversion of a patient's responses to the 5-item descriptive system of the EQ-5D-5L to a single index value,${ }^{26}$ were used to determine the utility value of each health state. The five items of the EQ-5D-5L assess mobility, self-care, usual activities, pain/discomfort, and anxiety/ depression, each of which is rated on a 5-point scale. ${ }^{25}$ Using a regression equation including all combinations of pain NRS score and sex, and average age of all patients (64.0 years), utility values for each pain NRS score per sex were computed. After calculating the weighted-average utility values for each pain NRS score using a sex ratio of the Japanese population at an average age, the average utility values for each pain severity category were computed as follows: 0.854 for no/mild pain, 0.738 for moderate pain, and 0.622 for severe pain (Table 1). Based on the utility values, a disutility value of transitioning to a more severe pain category was determined as -0.116 .

\section{Cost-Effectiveness}

Using the estimated costs and QALYs, the ICER was calculated using the following formula:

$$
\text { ICER }=\frac{\text { Costs pregabalin group }- \text { Costs usual care group }}{\text { QALYs pregabalin group }- \text { QALYs usual care group }}
$$

\section{Scenario And Sensitivity Analyses}

To assess the robustness of the results, scenario and sensitivity analyses were conducted from the healthcare payer's perspective. In the scenario analyses, the model assumed 
different scenarios from the base case: 1) an alternative initial distribution of severity categories (all patients started with moderate pain, or all started with severe pain), and 2) a different time horizon (3 or 24 months).

The uncertainties of the model parameters were also assessed through a series of one-way sensitivity analyses, by varying key variables using the $95 \%$ confidence intervals (CIs). The parameters considered included utility, disutility for transitioning to a more severe pain category, initial distribution probability of pain severity categories, discontinuation rates, transition probability of pain severity categories, pregabalin costs, other pain medication costs, and physical therapy costs. Furthermore, a probabilistic sensitivity analysis was also performed, in which cost, utility, and probability parameters were simultaneously varied by randomly sampling these values, per model iteration, from the distribution assigned for each parameter for 10,000 iterations. Cost parameters were assigned a gamma distribution, and utility and probability parameters were assigned a beta distribution. The ranges and distributions of disutilities in these sensitivity analyses were calculated based on the bootstrapping method, with consideration of co-variance between intercept and coefficient of pain NRS scores in the regression analysis using the Cholesky decomposition method. The obtained 10,000 pairs of incremental costs and QALYs based on probabilistic sensitivity analysis were plotted on a cost-effectiveness plane.

\section{Results}

\section{Pain Severity}

Figure 3 shows the simulated results of the distribution of patients with each pain severity category for the initial two months. A greater increase in the proportion of patients with no/mild pain was shown in patients receiving pregabalin-containing treatments than in patients receiving usual care alone, with $41.6 \%$ and $14.5 \%$ of patients in respective groups resulting in having no/mild pain after 8 weeks.

\section{Base Case Analyses}

The estimations of the costs and QALYs for base case scenarios are presented in Table 2. In the analysis from the payer's perspective, pregabalin-containing treatments resulted in a higher QALY than usual care alone (by 0.036), with additional costs of JPY 35,350, primarily for pregabalin acquisition. This resulted in the ICER of JPY 970,314 per QALY gained. The analyses from the societal perspective also considered productivity losses (both absenteeism and presenteeism). The productivity losses were JPY 18,653 less in the pregabalin group than in the usual care group, which largely offset the pregabalin costs, and resulted in the ICER of JPY 458,307 per QALY gained.

\section{Scenario And Sensitivity Analyses}

The results of the scenario analyses are summarized in Table 3. When assuming that all patients initially had moderate pain or

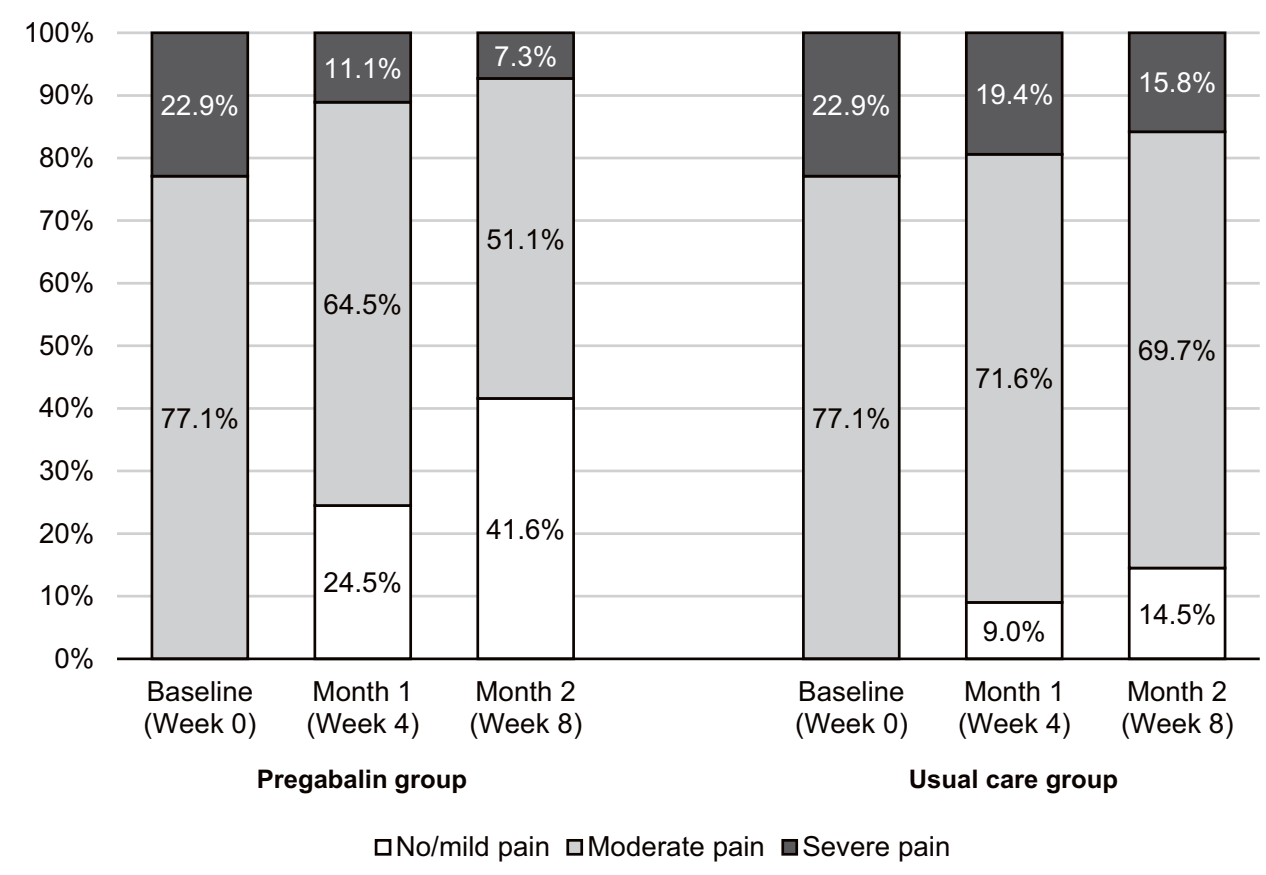

Figure 3 Simulated distribution of patient pain severity at baseline, Month I, and Month 2. 
Table 2 Base Case Analyses From The Perspectives Of Both The Healthcare Payer And Society

\begin{tabular}{|c|c|c|c|c|c|c|c|}
\hline \multirow[t]{3}{*}{ Perspectives } & \multicolumn{5}{|l|}{ Costs (JPY) } & \multirow[t]{3}{*}{ QALYs } & \multirow{3}{*}{$\begin{array}{l}\text { ICER (JPYI } \\
\text { QALY) }\end{array}$} \\
\hline & \multicolumn{3}{|c|}{ Medical Costs } & \multirow{2}{*}{$\begin{array}{l}\text { Productivity } \\
\text { Losses }\end{array}$} & \multirow[t]{2}{*}{ Total } & & \\
\hline & Pregabalin & $\begin{array}{l}\text { Other Pain } \\
\text { Medications }\end{array}$ & $\begin{array}{l}\text { Physical } \\
\text { Therapy }\end{array}$ & & & & \\
\hline Healthcare payer's perspective & & & & & & & \\
\hline Pregabalin group & 39,954 & 12,724 & 9,101 & - & 61,779 & 0.763 & - \\
\hline Usual care group & 0 & 13,996 & 12,432 & - & 26,428 & 0.727 & - \\
\hline Difference & 39,954 & $-1,272$ & $-3,331$ & - & 35,350 & 0.036 & 970,314 \\
\hline \multicolumn{8}{|c|}{$\begin{array}{l}\text { Societal perspective (absenteeism + } \\
\text { presenteeism) }\end{array}$} \\
\hline Pregabalin group & 39,954 & 12,724 & 9,101 & 658,736 & 720,515 & 0.763 & - \\
\hline Usual care group & 0 & 13,996 & 12,432 & 677,390 & 703,818 & 0.727 & - \\
\hline Difference & 39,954 & $-1,272$ & $-3,331$ & $-18,653$ & 16,697 & 0.036 & 458,307 \\
\hline
\end{tabular}

Notes: JPY $100=$ USD 0.89. Costs were rounded and displayed as integers.

Abbreviations: QALY, quality-adjusted life-year; ICER, the incremental cost-effectiveness ratio.

Table 3 Scenario Analyses With Assumptions Of Alternative Initial Pain Severity Distribution And Time Horizon (Healthcare Payer's Perspective)

\begin{tabular}{|l|l|l|l|}
\hline Scenarios & Total Medical Costs (JPY) & QALYs & ICER (JPY/QALY) \\
\hline Initial pain severity: All moderate & & & \\
Pregabalin group & 60,750 & 0.77 I & - \\
Usual care group & 26,428 & 0.739 & - \\
Difference & 34,322 & 0.032 & $1,073,627$ \\
\hline Initial pain severity: All severe & & & - \\
Pregabalin group & 65,242 & 0.738 & - \\
Usual care group & 26,428 & 0.687 & 754,236 \\
Difference & 38,814 & 0.051 & \\
\hline Time horizon: 3 months & & & - \\
Pregabalin group & 16,011 & 0.186 & - \\
Usual care group & 6,571 & 0.180 & $1,657,158$ \\
Difference & 9,440 & 0.0057 & \\
\hline Time horizon: 24 months & & & \\
Pregabalin group & 122,802 & 1,534 & \\
Usual care group & 52,905 & 1,456 & - \\
Difference & 69,897 & 0.077 & 902,835 \\
\hline
\end{tabular}

Note: JPY $100=$ USD 0.89

Abbreviations: QALY, quality-adjusted life year; ICER, incremental cost-effectiveness ratio.

all had severe pain, the analyses respectively resulted in the ICERs of JPY 1,073,627 and JPY 754,236 per QALY gained; both did not greatly deviate from the base case. The ICER varied more when an alternative time horizon was assumed, and resulted in the increased ICER of JPY 1,657,158 per QALY gained when the time horizon was shortened to 3 months.

The one-way sensitivity analyses demonstrated quite small variations in the ICERs for various costs, utility, and probability parameters, with the ICERs varying within the narrow range from JPY 800,000 to JPY 1,200,000 per QALY gained, even for the top 5 influential parameters (Figure 4). The highest ICER of JPY 1,151,479 per QALY gained was observed when disutility between no/mild pain and moderate pain was varied, and yet it was still similar to the result of the base case (JPY 970,314 per QALY gained).

Figure 5 presents the scatter plot of 10,000 pairs of incremental costs and QALYs based on probabilistic 


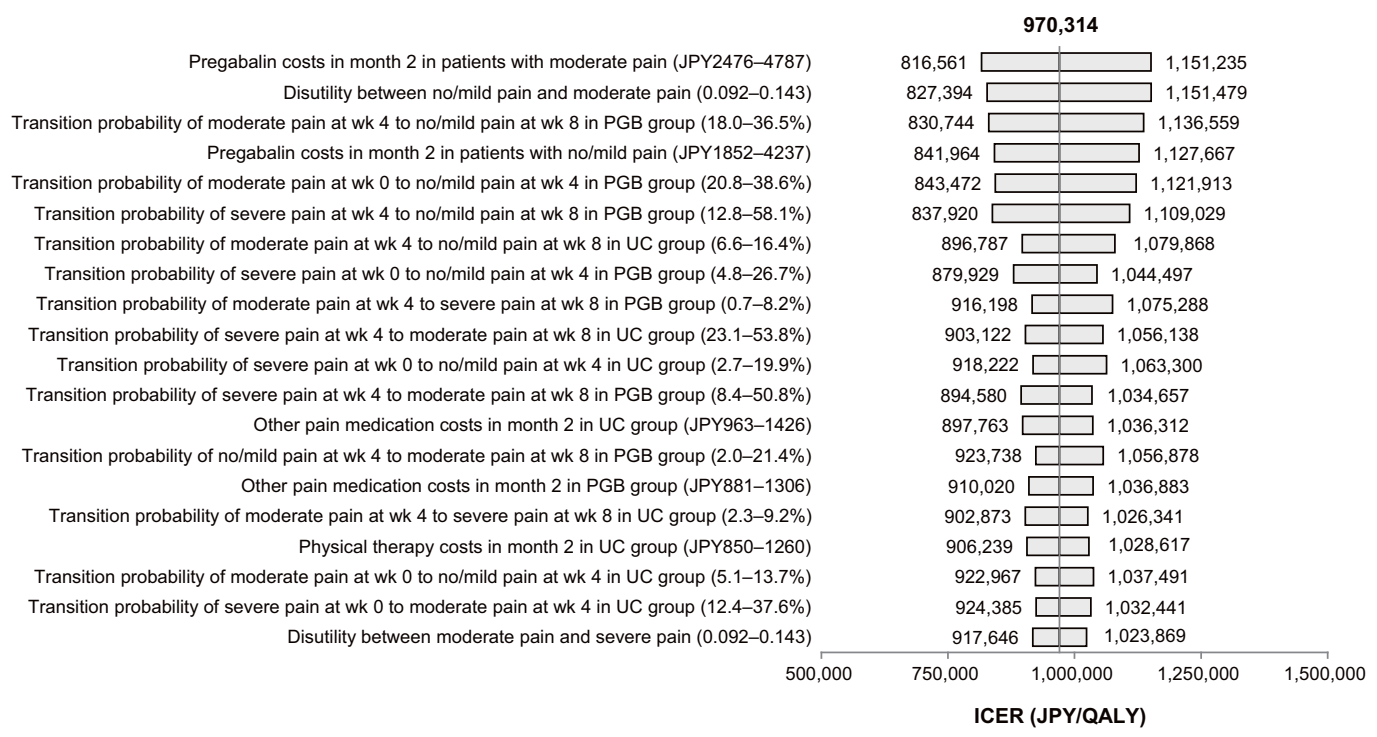

Figure 4 Tornado diagram of the 20 most sensitive parameters in one-way sensitivity analyses.

Abbreviations: wk, week; PGB group, pregabalin group; UC group, usual care group; ICER, incremental cost-effectiveness ratio; QALY, quality-adjusted life year.

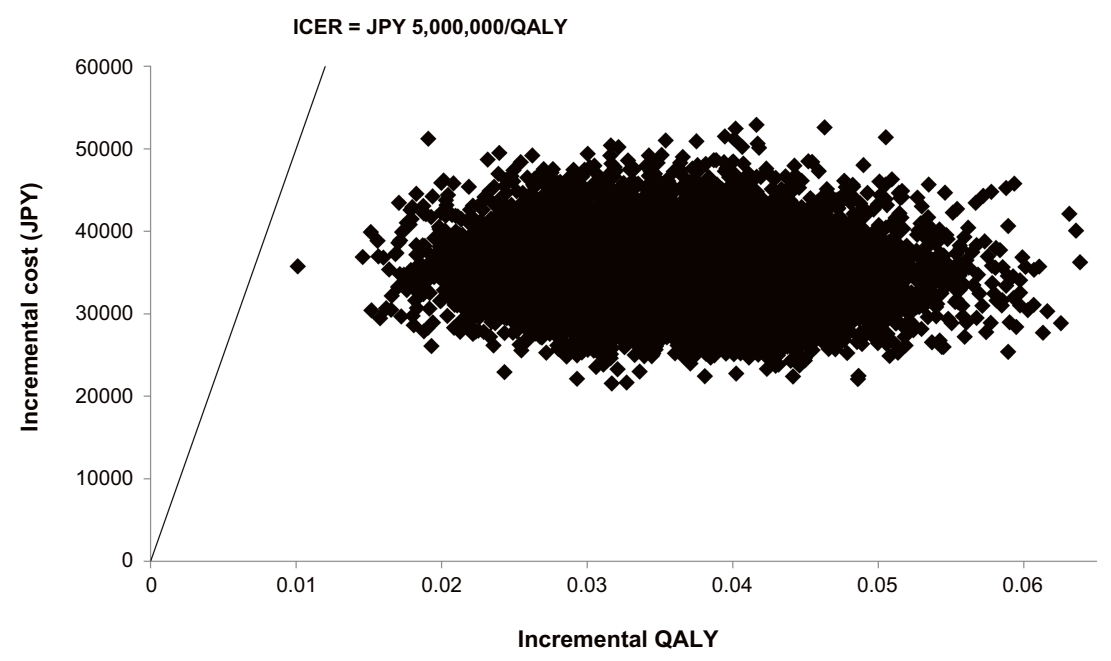

Figure 5 Scatter plot of pairs of incremental QALYs and costs.

Abbreviations: QALY, quality-adjusted life year; ICER, the incremental cost-effectiveness ratio.

sensitivity analysis. This cost-effectiveness plane showed that all samples were in the upper right quadrant with relatively small dispersion. The probability that pregabalin-containing treatments are cost-effective was $100 \%$ at a hypothetical threshold value of one additional QALY of JPY $5,000,000 .^{27}$

\section{Discussion}

In Japan, the indication of pregabalin has expanded since its launch in $2010,{ }^{12}$ and it has become widely used among people with various $\mathrm{NeP}$ conditions, including those associated with cervical radiculopathy and cervical myelopathy.
To our knowledge, this was the first cost-effectiveness analysis of pregabalin for the treatment of chronic cervical pain with a $\mathrm{NeP}$ component, and the results showed that using pregabalin, either alone or in addition to other analgesics, was cost-effective for the treatment of patients with this NeP condition in Japan, compared with pain treatment only with conventional analgesics.

From the payer's perspective, the estimated ICER was JPY 970,314 per QALY gained, which was lower than those in previous studies reporting the cost-effectiveness of pregabalin for refractory or peripheral NeP. ${ }^{15,28,29}$ From the societal perspective, the ICER was substantially lowered to JPY 
458,307 per QALY gained because the reduced productivity losses by pregabalin-containing treatments offset the pregabalin costs by almost half. In patients with chronic pain, presenteeism greatly contributes to their productivity losses and causes a substantial economic burden on patients. ${ }^{30,31} \mathrm{~A}$ Japanese study previously reported that among 34 health conditions, neck pain or stiff shoulders contributed the most to costs of presenteeism in Japan. ${ }^{32}$ The use of pregabalin for the management of patients with neck pain with a NeP component may serve to reduce some of this large burden.

The scenario and sensitivity analyses demonstrated the robustness of the result of the base case analysis. The ICER was somewhat sensitive to the time horizon, which resulted in the increased ICER of 1,657,158 per QALY gained within the 3-month time horizon. However, the gap with the base case was no more than JPY 700,000 per QALY gained, and the value is well below the estimated willingness to pay for one additional QALY in Japan (JPY $5,000,000-6,750,000) .{ }^{27,33}$ Additionally, one-way sensitivity analyses showed that ICERs varied within only a narrow range, which indicates the stability of ICERs for various costs, utility, and probabilistic parameters. Furthermore, our probabilistic sensitivity analysis demonstrated that if the hypothetical threshold value of JPY $5,000,000$, based on the above-mentioned willingness to pay in Japan, is an appropriate threshold value for one additional QALY, pregabalin-containing treatments would be cost-effective with a probability of $100 \%$. As the ICERs estimated in this analysis are also well below the commonly-referenced thresholds in other countries, such as EUR 20,000-30,000 in the UK $(\text { EUR 1.0 = JPY 131.11) })^{34}$ and USD 50,000-100,000 in the US (USD 1.0 = JPY 112.32), ${ }^{35}$ pregabalin-containing treatments can be considered as highly cost-effective for this NeP condition in Japan.

In the base case, starting pregabalin resulted in 0.036 higher QALY gain than usual care alone, probably reflecting the favorable effects of pregabalin for improvement of pain, function, and sleep disturbance. ${ }^{14,36}$ Although the observed QALY gain seemed rather small, this may be in part because of the non-fatal nature of the condition and the short time horizon of the model. However, the potential clinical benefits of pregabalin are apparent from the results of our simulation showing the greater increase in the proportion of patients with no or mild pain after two months $(+41.6 \%)$ than in usual care alone $(+14.5 \%)$ (see Figure 3$)$. The proportion of patients with severe pain would be reduced more greatly with pregabalin-containing treatments in two months than with usual care alone
$(-15.6 \%$ versus $-7.1 \%)$. These results suggest a substantial clinical benefit of pregabalin-containing treatments for this patient population. In this analysis, we did not go further in examining which component of QOL (whether improvement of physical function, alleviation of pain, or change in mental state) contributed the most to the QALY gain. These topics are worth investigating in future studies to obtain a deeper understanding of the clinical benefits of pregabalin.

Comparing our results with a previous parallel costeffectiveness analysis of pregabalin for chronic low back pain with a NeP component ${ }^{16}$ implied a difference in potential treatments between the two pain conditions. Despite similar QALYs of the pregabalin groups in two analyses (cervical pain: 0.763 versus low back pain: 0.766), the incremental QALYs gained by pregabalin-containing treatments were larger in the present analysis than in the low back pain analysis ( 0.036 versus 0.014 , respectively), reflecting the slightly lower QALYs in the usual care group in the present analysis $(0.727$ versus 0.752 , respectively). These results suggest that patients with chronic cervical pain with a NeP component may be more difficult to manage without pregabalin, compared with those with low back pain with a NeP component. One possible explanation is that relative to chronic low back pain, which often involves both nociceptive and neuropathic pain mechanisms (that is, mixed pain ${ }^{37}$ ) for which a combination of medications for both pain types is suggested, ${ }^{38,39}$ mixed pain may be less likely among patients with chronic cervical pain, and thus there may have been fewer patients who benefited from medications for nociceptive pain such as NSAIDs. Considering these, once the involvement of $\mathrm{NeP}$ is identified in patients with chronic neck pain, physicians may first need to consider prescribing pregabalin because otherwise, pain control may be difficult to achieve.

One major strength of this cost-effectiveness analysis lies in the use of patient-level outcome data from a realworld clinical setting, which contributed to a better generalization of the results to routine clinical practice in Japan. However, this analysis also has several limitations. In the observational study, to avoid bias in patients included in each treatment group, patients were enrolled after they were prescribed pain medications, and all eligible patients who agreed to participate during the 1-year enrollment period were enrolled. Despite these efforts, because all treatment choices were made by physicians based on their clinical judgment, the potential for bias 
still cannot be eliminated; for example, physicians may have been more likely to prescribe pregabalin to patients with greater pain, which was suggested by the slightly higher pain NRS scores at baseline in the pregabalin group. Given that pregabalin was more commonly used among patients with severe pain, which may be more difficult to control than mild pain, the cost-effectiveness of pregabalin-containing treatments may be underestimated in this analysis. As another example, the pregabalin group contained more male and younger patients than the usual care group, probably because physicians avoided prescribing pregabalin to frail elderly patients in consideration of the potential risks of falls and fractures associated with pregabalin's adverse effects such as dizziness. In this analysis, however, to estimate the improvement of pain by each treatment, we set the distributions of pain severity and QOL at baseline to be the same in the two groups. Also, the propensity for pain improvement does not depend on age and sex. Therefore, we reasoned that the age and sex differences would not affect the results of this analysis. Another limitation is that medical costs included only limited elements and not all healthcare costs (such as surgery or hospitalization costs) were considered in this analysis, on the assumption that the cases potentially requiring such additional healthcare utilization were expected to be equally rare in both groups. The observational study enrolled patients in stable conditions; thus, surgery or hospital admission for their symptoms of pain would not be anticipated in these patients, irrespective of group. Additionally, this analysis did not consider treatment costs for adverse events of pregabalin because additional treatments are not required for the management of its common adverse events such as dizziness and somnolence; these adverse events are managed by discontinuing pregabalin even when they are serious, although they are mostly non-serious. Thus, we posit that including some additional medical costs would not likely result in a tremendous rise in the ICER and reach an opposite conclusion; nevertheless, we should acknowledge that an analysis which included these costs would result in a different ICER. Third, we calculated work productivity losses using the WPAI, but the calculation method cannot account for the work productivity loss of individuals who do not have a paid job, meaning that a loss of work productivity of someone without a job because of severe pain was not included in our calculations. As such, the estimated costs of productivity losses depend on the estimation method, which should be considered when interpreting these results.

\section{Conclusion}

For the first time, this analysis provided evidence that using pregabalin, either alone or along with other analgesics, was cost-effective for the treatment of patients with chronic cervical pain with a NeP component in Japan, with the ICERs well below the commonly-referenced thresholds for cost-effectiveness. The cost-effectiveness of pregabalin was further strengthened when productivity losses (both absenteeism and presenteeism) were included. The results of this analysis underscore the benefits of pregabalin for this $\mathrm{NeP}$ condition, which may help pregabalin become a more common treatment option in the management of these patients.

\section{Acknowledgment}

Pfizer Japan sponsored this study.

\section{Disclosure}

Nozomi Ebata, Kazutaka Nozawa, and Koichi Fujii are employees of Pfizer Japan Inc. Tatsunori Murata and Shigeki Zeniya are employees of CRECON Medical Assessment Inc. Yuri Haga is an employee of Clinical Study Support, Inc. Manabu Akazawa, Ataru Igarashi, and Toshihiko Taguchi were not financially compensated for their collaboration in this project or for the development of this manuscript. Manubu Akazawa reports personal fees from Takeda and Pfizer, outside the submitted work. Ataru Igarashi reports grants and non-financial support from Pfizer Inc., during the conduct of the study. He also reports grant and personal fees from Gilead Sciences K.K, CSL Behring Japan Inc, Fuji film Inc Milliman Inc., Pfizer Inc., Novartis, Astellas Pharma Inc., CreativCeuticals Inc, Takeda Pharmaceuticals Inc, Novo Nordisc Inc., Intuitive Surgical G.K., Boston scientific Japan Inc., Nippon Boeringer Ingelheim Inc., Eli Lilly Japan K.K., Edwards Life Science Inc., and non-financial support from Terumo corporation, outside the submitted work. The authors report no other conflicts of interest in this work.

\section{References}

1. GBD. 2015 disease and injury incidence and prevalence collaborators Global, regional, and national incidence, prevalence, and years lived with disability for 310 diseases and injuries, 1990-2015: a systematic analysis for the Global Burden of Disease Study 2015. Lancet. 2016;388(10053):1545-1602. 
2. Takasawa E, Yamamoto A, Kobayashi T, et al. Characteristics of neck and shoulder pain in the Japanese general population. $J$ Orthop Sci. 2015;20(2):403-409. doi:10.1007/s00776-014-0676-2

3. Nakamura M, Nishiwaki Y, Ushida T, Toyama Y. Prevalence and characteristics of chronic musculoskeletal pain in Japan. J Orthop Sci. 2011;16(4):424-432. doi:10.1007/s00776-011-0102-y

4. Cohen SP. Epidemiology, diagnosis, and treatment of neck pain. Mayo Clin Proc. 2015;90(2):284-299. doi:10.1016/j.mayocp.2014. 12.011

5. Torrance N, Smith BH, Bennett MI, Lee AJ. The epidemiology of chronic pain of predominantly neuropathic origin. Results from a general population survey. J Pain. 2006;7(4):281-289. doi:10.1016/ j.jpain.2005.11.008

6. Taylor RS. Epidemiology of refractory neuropathic pain. Pain Pract. 2006;6(1):22-26. doi:10.1111/j.1533-2500.2006.00054.x

7. Liu R, Kurihara C, Tsai HT, et al. Classification and treatment of chronic neck pain: a longitudinal cohort study. Reg Anesth Pain Med. 2017;42(1):52-61. doi:10.1097/AAP.0000000000000615

8. Attal N, Cruccu G, Baron R, et al. EFNS guidelines on the pharmacological treatment of neuropathic pain: 2010 revision. Eur J Neurol. 2010;17(9):1113-1123. doi:10.1111/j.1468-1331.2010.02999.x

9. Dworkin RH, O'Connor AB, Backonja M, et al. Pharmacologic management of neuropathic pain: evidence-based recommendations. Pain. 2007;132(3):237-251. doi:10.1016/j.pain.2007.08.033

10. Sumitani M, Sakai T, Matsuda Y, et al. Executive summary of the clinical guidelines of pharmacotherapy for neuropathic pain: second edition by the Japanese Society of Pain Clinicians. J Anesth. 2018;32 (3):463-478. doi:10.1007/s00540-018-2501-0

11. LYRICA $^{\circledR}$ (pregabalin) [prescribing information]. Available from: http://labeling.pfizer.com/ShowLabeling.aspx?format=PDF\&id=561. Accessed June 27, 2018.

12. Eisai Corporate Website. New indication approved in Japan for lyrica ${ }^{\circledR}$ capsules. Available from: https://www.eisai.com/news/ news201311.html. Accessed June 18, 2018.

13. European Medicines Agency. Lyrica: EPAR-product information. Available from: https://www.ema.europa.eu/documents/product-infor mation/lyrica-epar-product-information_en.pdf. Accessed November 22, 2018

14. Taguchi T, Nozawa K, Parsons B, et al. Effectiveness of pregabalin for treatment of chronic cervical radiculopathy with upper limb radiating pain: an 8-week, multicenter prospective observational study in Japanese primary care settings. J Pain Res. 2019;12:14111424. doi:10.2147/JPR.S191906

15. Ikeda S, Ogawa S, Hosokawa T, et al. Cost-effectiveness analysis of pregabalin for treatment of peripheral neuropathic pain. Jpn $J$ Pharmacoepidemiol. 2011;16(1):1-9. Japanese. doi:10.3820/jjpe.1 6.1

16. Igarashi A, Akazawa M, Murata T, et al. Cost-effectiveness analysis of pregabalin for treatment of chronic low back pain in patients with accompanying lower limb pain (neuropathic component) in Japan. Clinicoecon Outcomes Res. 2015;7:505-520.

17. Satoh J, Yagihashi S, Baba M, Suzuki M, Arakawa A, Yoshiyama T. Efficacy and safety evaluation of pregabalin treatment over 52 weeks in patients with diabetic neuropathic pain extended after a doubleblind placebo-controlled trial. J Diabetes Investig. 2011;2(6):457463. doi:10.1111/j.2040-1124.2011.00122.x

18. Onouchi K, Koga H, Yokoyama K, Yoshiyama T. An open-label, long-term study examining the safety and tolerability of pregabalin in Japanese patients with central neuropathic pain. J Pain Res. 2014;7:439-447.

19. The Japanese Orthopaerdic Association. Japanese Orthopaerdic Association (JOA) Clinical Practice Guideline on the Cervical Spondylotic Myelopathy 2015. 2nd ed. Tokyo: Nankodo; 2015. Japanese.
20. Kavanagh RG, Butler JS, O'Byrne JM, Poynton AR. Operative techniques for cervical radiculopathy and myelopathy. Adv Orthop. 2012;2012:794087. doi:10.1155/2012/794087

21. Iyer S, Kim HJ. Cervical radiculopathy. Curr Rev Musculoskelet Med. 2016;9(3):272-280. doi:10.1007/s12178-016-9349-4

22. Reilly Associates [homepage on the Internet]. WPAI: GH V2.0. Margaret Reilly Associates, Inc.; 2002 [updated October 20, 2004]. Available from: http://www.reillyassociates.net/WPAI_GH.html. Accessed June 18, 2018.

23. Lofland JH, Pizzi L, Frick KD. A review of health-related workplace productivity loss instruments. Pharmacoeconomics. 2004;22(3):165184. doi:10.2165/00019053-200422030-00003

24. Ministry of Health, Labour and Welfare. Basic survey on wage structure in 2016 (in Japanese). Available from: https://www.mhlw.go.jp/toukei/ itiran/roudou/chingin/kouzou/z2016/index.html. Accessed December $25,2018$.

25. Herdman M, Gudex C, Lloyd A, et al. Development and preliminary testing of the new five-level version of EQ-5D (EQ-5D-5L). Qual Life Res. 2011;20(10):1727-1736. doi:10.1007/s11136-011-9903-x

26. Ikeda S, Shiroiwa T, Igarashi A, et al. Developing a Japanese version of the EQ-5D-5L value set. J Nat Inst Public Health. 2015;64(1):4755. Japanese.

27. Shiroiwa T, Sung YK, Fukuda T, Lang HC, Bae SC, Tsutani K. International survey on willingness-to-pay (WTP) for one additional QALY gained: what is the threshold of cost effectiveness? Health Econ. 2010;19(4):422-437. doi:10.1002/hec.1481

28. Prettyjohns M, Sandelin R, Lister S, Norrefalk JR. A cost-utility study of the use of pregabalin added to usual care in refractory neuropathic pain patients in a Swedish setting. $J$ Med Econ. 2012;15(6):1097-1109. doi:10.3111/13696998.2012.704458

29. Gordon J, Lister S, Prettyjohns M, McEwan P, Tetlow A, Gabriel Z. A cost-utility study of the use of pregabalin in treatment-refractory neuropathic pain. J Med Econ. 2012;15(2):207-218. doi:10.3111/ 13696998.2011.632797

30. Takura T, Ushida T, Kanchiku T, et al. The societal burden of chronic pain in Japan: an internet survey. J Orthop Sci. 2015;20(4):750-760. doi:10.1007/s00776-015-0730-8

31. Montgomery W, Sato M, Nagasaka Y, Vietri J. The economic and humanistic costs of chronic lower back pain in Japan. Clinicoecon Outcomes Res. 2017;9:361-371. doi:10.2147/CEOR.S134130

32. Nagata T, Mori K, Ohtani M, et al. Total health-related costs due to absenteeism, presenteeism, and medical and pharmaceutical expenses in Japanese employers. J Occup Environ Med. 2018;60(5):e273e280. doi:10.1097/JOM.0000000000001291

33. Ohkusa Y, Sugawara T. Research for willingness to pay for one QALY gain. J Health Care Soc. 2006;16(2):157-165. Japanese. doi:10.4091/iken.16.157

34. National Institute for Health and Care Excellence (NICE). Guide to the methods of technology appraisal; 2013. Available from: https://www. nice.org.uk/process/pmg9/chapter/foreword. Accessed June 18, 2018.

35. Neumann PJ, Cohen JT, Weinstein MC. Updating cost-effectivenessthe curious resilience of the \$50,000-per-QALY threshold. $N$ Engl $J$ Med. 2014;371(9):796-797. doi:10.1056/NEJMoa1410490

36. Taguchi T, Igarashi A, Watt $\mathrm{S}$, et al. Effectiveness of pregabalin for the treatment of chronic low back pain with accompanying lower limb pain (neuropathic component): a non-interventional study in Japan. J Pain Res. 2015;8:487-497.

37. Freynhagen R, Baron R. The evaluation of neuropathic components in low back pain. Curr Pain Headache Rep. 2009;13(3):185-190. doi:10.1007/s11916-009-0032-y

38. Romanò CL, Romanò D, Bonora C, Mineo G. Pregabalin, celecoxib, and their combination for treatment of chronic low-back pain. J Orthop Traumatol. 2009;10(4):185-191. doi:10.1007/s10195-009-0077-z

39. Morlion B. Pharmacotherapy of low back pain: targeting nociceptive and neuropathic pain components. Curr Med Res Opin. 2011;27 (1):11-33. doi:10.1185/03007995.2010.534446 


\section{Publish your work in this journal}

The Journal of Pain Research is an international, peer reviewed, open access, online journal that welcomes laboratory and clinical findings in the fields of pain research and the prevention and management of pain Original research, reviews, symposium reports, hypothesis formation and commentaries are all considered for publication. The manuscript management system is completely online and includes a very quick and fair peer-review system, which is all easy to use. Visit http:// www.dovepress.com/testimonials.php to read real quotes from published authors.

Submit your manuscript here: https://www.dovepress.com/journal-of-pain-research-journal 